\title{
Stability of pear-shaped configurations bifurcated from a pressurized spherical balloon
}

\author{
Y.B. $\mathrm{Fu}^{1,2 *}$ and Y.X. $\mathrm{Xie}^{2,3}$ \\ ${ }^{1}$ Department of Mathematics, Keele University, ST5 5BG, U.K. \\ ${ }^{2}$ Department of Mechanics, Tianjin University, Tianjin 300072, China \\ ${ }^{3}$ Tianjin Key Laboratory of Modern Engineering Mechanics, Tianjin 300072, China
}

January 26, 2014

\begin{abstract}
It is well-known that for most spherical rubber balloons the pressure versus volume curve associated with uniform inflation has an $N$-shape (the pressure increases rapidly to a maximum, falls to a minimum, and subsequently increases monotonically), and that somewhere along the descending branch of this curve the spherical shape may bifurcate into a pear shape through localized thinning near one of the poles. The bifurcation is associated with the (uniform) surface tension reaching a maximum. It is previously known that whenever a pear-shaped configuration becomes possible, it has lower energy than the co-existing spherical configuration, but the stability of the pear-shaped configuration itself is unknown. With the use of the energy stability criterion, it is shown in this paper that the pear-shaped configuration is unstable under pressure control, but stable under mass control. Our calculations are carried out using the Ogden material model as an example, but it is expected that the qualitative stability results should also be valid for other material models that predict a similar $N$-shaped behaviour for uniform inflation.
\end{abstract}

\section{1 introduction}

Inflating a membrane balloon is a classical problem in Finite Elasticity (Adkins and Rivlin 1952), which has been studied from a variety of perspectives; see, e.g., Crisp and HartSmith (1971), Sagiv (1990), De Tommasi et al (2013), and the references therein. For most spherical rubber balloons, the pressure versus volume curve associated with uniform inflation has an $\mathrm{N}$-shape, and it is well-known that somewhere along the descending

\footnotetext{
${ }^{*}$ Corresponding author, Email: y.fu@keele.ac.uk
} 
branch of this curve (see Fig. 1) the spherical shape may bifurcate into a pear shape through localized thinning near one of the poles; see Feodosev (1968), Needleman (1977), Haughton and Ogden (1978), Haughton (1980), and Chen and Healey (1991). It was shown by Chen and Healey (1991) that whenever the pear-shaped configuration is possible, it has lower energy than the co-existing spherical configuration. This implies, via the energy stability criterion, that the co-existing spherical configuration is unstable, but it does not give any information about the stability of the pear-shaped configuration. Over the remaining section of the descending curve where the pear-shaped configuration is not possible, stability of the spherical configuration has only been studied with respect to spherical perturbations, and it is thought that the spherical configuration is unstable under pressure control but stable under mass control (Alexander 1971). There also exists a number of studies on the inflation and stability of multi-lobed spherical balloons (see, e.g., Crisp and Hart-Smith 1971, Müler and Struchtrup 2002), but again, stability is only considered with respect to spherical perturbations.

In this paper, we fill a gap in the literature by studying the stability of both spherical and pear-shaped solutions on the above-mentioned descending branch with respect to axi-symmetric perturbations. In addition to its intrinsic theoretical value, the present study may have some relevance to the mathematical modelling of the initiation and final rupture of saccular aneurysms in human arteries (Austin et al. 1989). We also observe that pressurized balloons are increasingly used in a variety of engineering situations, ranging from extra-terrestrial use as foldable habitats (Jenkins 2001) to microelectromechanical systems as actuators (Youda and Konishi 2002, Keplinger et al 2012, Rudykha et al 2012). Such applications invariably require a good understanding of their stability properties.

Since the inflation problem under consideration is conservative, the main method that we use to assess stability is the energy criterion although its connection with the spectral method is also discussed. More precisely, we calculate the minimum of the second variation of the total energy with respect to all kinematically admissible axi-symmetric perturbations. An inflated configuration is said to be stable if this minimum is positive, and unstable if it is negative. It is generally recognized that the energy stability criterion should be used with caution. For conservative systems, it is known that under appropriate assumptions, the solution being an energy minimizer implies (nonlinear) stability in the Liapunov sense (van der Heijden 2009), but the converse has not been rigorously proved. In contrast, the spectral method is a very effective method for predicting linear instability. We shall show in this paper that if the above-mentioned minimum is negative, then linear (spectral) instability is implied. For a more rigorous discussion of stability criteria for nonlinear elasticity, we refer to the book by Marsden and Hughes (1993) and the more recent article by Knops (2001).

The rest of this paper is divided into four sections as follows. After formulating the 


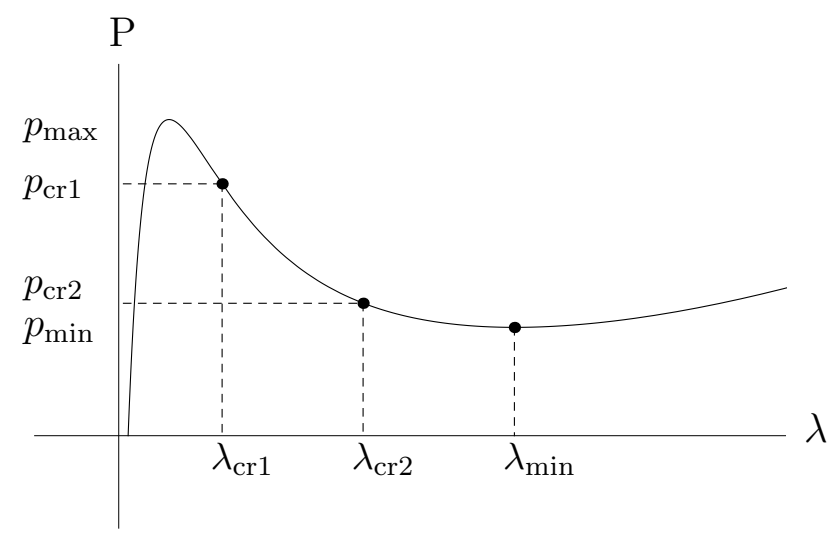

Figure 1: A typical profile of pressure $P$ versus principal stretch $\lambda$ in uniform inflation of a spherical shell. Internal volume is proportional to $\lambda^{3}$

inflation problem, we explain in Section 2 a procedure that can be used to find all axisymmetric solutions (spherical or pear-shaped in particular) whenever they exist. We then examine in Section 3 the stability, under pressure control, of both the spherical and pearshaped solutions using a combination of the spectral method and the energy criterion. In Section 4, stability of the pear-shaped solution under mass control is studied. The paper is concluded in the final section with further discussions.

\section{Pear-shaped configurations bifurcated from spher- ical configurations}

We consider a spherical balloon that is described by

$$
R(\theta)=\sin \theta, \quad Z(\theta)=1-\cos \theta, \quad 0 \leq \theta \leq \pi
$$

in terms of cylindrical polar coordinates $(R, \theta, Z)$ in its undeformed configuration. Without loss of generality, we have assumed the constant radius to be unity, which is equivalent to using the radius as the unit for length.

We focus on axisymmetric deformations described by

$$
r=r(\theta), \quad z=z(\theta)
$$

where $(r, \theta, z)$ are cylindrical polar coordinates in the deformed configuration. This form includes uniformly inflated solutions and pear-shaped bifurcated solutions. Denote by $d S$ and $d s$ the arclengths measured from $\theta=0$ in the undeformed and deformed configurations, respectively. We then have $d S=d \theta$ and $d s=\sqrt{r^{\prime 2}+z^{\prime 2}} d \theta$, where a prime denotes differentiation with respect to $\theta$. The principal directions of stretch coincide with the lines of latitude, the meridian and the normal to the deformed surface. Thus, the principal 
stretches are given by

$$
\lambda_{1}=\frac{r}{R}, \quad \lambda_{2}=\frac{d s}{d S}=\sqrt{r^{\prime 2}+z^{\prime 2}}, \quad \lambda_{3}=\frac{h}{H},
$$

where $H$ and $h$ are the undeformed and deformed thicknesses, respectively.

By considering equilibrium of an infinitesimal volume element in the 1- and 2- directions, respectively, we obtain (Haughton 1980)

$$
\begin{gathered}
r\left(h \sigma_{2}\right)^{\prime}+h r^{\prime}\left(\sigma_{2}-\sigma_{1}\right)=0, \\
\frac{z^{\prime} \sigma_{1}}{r \lambda_{2}}+\frac{\sigma_{2}\left(r^{\prime} z^{\prime \prime}-z^{\prime} r^{\prime \prime}\right)}{\lambda_{2}^{3}}=\lambda_{1} \lambda_{2} P,
\end{gathered}
$$

where $\sigma_{1}$ and $\sigma_{2}$ denote the principal Cauchy stresses, and $P$ is the actual internal pressure scaled by $\mathrm{H}$. The two equilibrium equations have one integral, which corresponds to the constancy (zero in this case) of the resultant force at any cross-section, and is given by

$$
z^{\prime}=\frac{\operatorname{Pr} \lambda_{1} \lambda_{2}^{2}}{2 \sigma_{2}}
$$

In particular, at any cross section where $r^{\prime}=0$, we obtain

$$
1=\frac{P R(\theta) \lambda_{1}^{2} \lambda_{2}}{2 \sigma_{2}}
$$

which can be used to express $\lambda_{2}$ in terms of $\lambda_{1}$ at that particular cross section.

In some papers the equilibrium equations are given in terms of the principal curvatures

$$
\kappa_{1}=\frac{\cos \phi}{r}, \quad \kappa_{2}=-\frac{d \phi}{d s}=\frac{(\cos \phi)^{\prime}}{\sin \phi \lambda_{2}}=\frac{r^{\prime} z^{\prime \prime}-z^{\prime} r^{\prime \prime}}{\lambda_{2}^{3}},
$$

where $\phi$ is the angle between the meridian and the $z$-axis. The equilibrium equations then take the alternative form

$$
\left(h \sigma_{2} r\right)^{\prime}=\left(h \sigma_{1}\right) r^{\prime}, \quad \kappa_{1}\left(h \sigma_{1}\right)+\kappa_{2}\left(h \sigma_{2}\right)=H P .
$$

With the use of the relations $r^{\prime}=\lambda_{2} \sin \phi, \quad z^{\prime}=\lambda_{2} \cos \phi$, the equilibrium equations can be rewritten as a system of first-order ordinary differential equations:

$$
\begin{aligned}
\lambda_{1}^{\prime} & =\frac{\lambda_{2} \sin \phi-\lambda_{1} \cos \theta}{\sin \theta}, \\
\lambda_{2}^{\prime} & =\frac{W_{1}-\lambda_{2} W_{12}}{W_{22}} \cdot \frac{\sin \phi}{\sin \theta}-\frac{W_{2}-\lambda_{1} W_{12}}{W_{22}} \cdot \cot \theta, \\
\phi^{\prime} & =\frac{W_{1}}{W_{2}} \frac{\cos \phi}{\sin \theta}-\frac{P \lambda_{1} \lambda_{2}}{W_{2}},
\end{aligned}
$$

where $W_{1}=\partial W / \partial \lambda_{1}, W_{12}=\partial^{2} W / \partial \lambda_{1} \partial \lambda_{2}$, and etc, and $W\left(\lambda_{1}, \lambda_{2}\right)=\tilde{W}\left(\lambda_{1}, \lambda_{2}, \lambda_{1}^{-1} \lambda_{2}^{-1}\right)$, $\tilde{W}$ being the three-dimensional strain-energy function (measured per unit volume in the undeformed configuration). In writing down the above equations, use has also been made 
of the constitutive relations $\sigma_{1}=\lambda_{1} W_{1}, \sigma_{2}=\lambda_{2} W_{2}$, and the assumption that the rubber material is incompressible. In our example calculations, we shall adopt the Ogden material model (Ogden 1972) given by

$$
\tilde{W}=\sum_{r=1}^{3} \tilde{\mu}_{r}\left(\lambda_{1}^{\alpha_{r}}+\lambda_{2}^{\alpha_{r}}+\lambda_{3}^{\alpha_{r}}-3\right) / \alpha_{r}
$$

where the material constants are given by $\alpha_{1}=1.3, \alpha_{2}=5.0, \alpha_{3}=-2.0, \tilde{\mu}_{1}=1.491, \tilde{\mu}_{2}=$ $0.003, \tilde{\mu}_{3}=-0.023$, and $\tilde{W}$ has been scaled by the shear modulus $\mu$ for infinitesimal deformations.

The system of equations (2.8) obviously admits a uniform solution given by

$$
\lambda_{1}=\lambda_{2}=\lambda, \quad \phi=\frac{\pi}{2}-\theta, \quad P=\frac{2 W_{2}(\lambda, \lambda)}{\lambda^{2}},
$$

where $\lambda$ is a constant. This corresponds to uniform inflation. For most rubber materials, and in particular for the Ogden material model given by (2.9), the dependence of pressure on the volume has an $N$ shape. An important question is under what conditions the uniform solution will bifurcate into a non-uniform solution. This question is now wellunderstood, and it is known that the bifurcation condition for a pear-shaped configuration to initiate is given by

$$
\frac{\lambda^{2}}{H} \frac{d\left(h \sigma_{1}\right)}{d \lambda}=\lambda^{2} \frac{d}{d \lambda}\left(\frac{W_{1}}{\lambda}\right)=\lambda\left(W_{11}+W_{12}\right)-W_{1}=0
$$

that is, when the membrane tension $h \sigma_{1}$ reaches a maximum. It can easily be shown that this condition cannot be satisfied before the pressure maximum is reached (Haughton and Ogden 1978). For the Ogden material, the bifurcation condition has two roots given by

$$
\lambda_{\mathrm{cr} 1}=1.7783, \quad \lambda_{\mathrm{cr} 2}=2.5137
$$

with the associated pressures

$$
P_{\mathrm{cr} 1}=1.1056, \quad P_{\mathrm{cr} 2}=0.7482
$$

where here and hereafter the pressure values have been scaled by $\mu H$. The stretch values corresponding to the pressure maximum $P_{\max }(=1.3013)$ and minimum $P_{\min }(=0.5662)$ are given by

$$
\lambda_{\max }=1.3744, \quad \lambda_{\min }=4.2729,
$$

respectively. Thus, as expected, we have $\lambda_{\max }<\lambda_{\text {cr1 }}<\lambda_{\mathrm{cr} 2}<\lambda_{\min }$; see Fig. 1 .

Non-uniform bifurcated solutions can be obtained numerically by integrating the system of equations (2.8) subject to appropriate boundary conditions. We note, however, that this system has a removable singularity at the two poles $\theta=0, \pi$. To avoid evaluation at the poles, we integrate from $\theta=\delta$ to $\theta=\pi-\delta$ instead, where $\delta$ is a sufficiently small 
constant. We then need sufficiently accurate estimates for the values of $\lambda_{1}, \lambda_{2}$ and $\phi$ at the poles.

Because of the axi-symmetry at the poles, we first have

$$
\lambda_{1}(0)=\lambda_{2}(0), \quad \lambda_{1}(\pi)=\lambda_{2}(\pi), \quad \phi(0)=\frac{\pi}{2}, \quad \phi(\pi)=-\frac{\pi}{2},
$$

and

$$
\lambda_{1}^{\prime}(0)=\lambda_{2}^{\prime}(0)=\phi^{\prime \prime}(0)=0 .
$$

On differentiating $(2.8)_{1,2}$ with respect to $\theta$ and then evaluating at the two poles, we obtain two linear equations for $\lambda_{1}^{\prime \prime}$ and $\lambda_{2}^{\prime \prime}$, the solution of which then gives

$$
\begin{array}{ll}
\lambda_{1}^{\prime \prime}=\frac{\left(3 \lambda_{1} W_{11}-\lambda_{1} W_{12}+W_{1}\right)\left(1-\phi^{2}\right)}{8 W_{11}}, & \theta=0, \pi, \\
\lambda_{2}^{\prime \prime}=\frac{\left(\lambda_{1}\left(W_{11}-3 W_{12}\right)+3 W_{1}\right)\left(1-\phi^{\prime 2}\right)}{8 W_{11}}, & \theta=0, \pi .
\end{array}
$$

Expanding $(2.8)_{3}$ at the two poles and solving the resulting equations, we obtain, at $\theta=0, \pi$

$$
\begin{gathered}
\phi^{\prime}=-\frac{P \lambda_{1}^{2}}{2 W_{2}} . \\
\phi^{\prime \prime \prime}=\frac{\lambda_{1} P}{64 W_{1}^{4} W_{11}}\left(P^{2} \lambda_{1}^{4}-4 W_{1}^{2}\right)\left\{\left(W_{11}-9 W_{12}\right) W_{1} \lambda_{1}+3\left(W_{12}^{2}-W_{11}^{2}\right) \lambda_{1}^{2}+6 W_{1}^{2}\right\} .
\end{gathered}
$$

We may then write

$$
\begin{aligned}
\lambda_{1}(\delta) & =\lambda_{1}(0)+\frac{1}{2} \lambda_{1}^{\prime \prime}(0) \delta^{2}+O\left(\delta^{4}\right), \\
\lambda_{2}(\delta) & =\lambda_{1}(0)+\frac{1}{2} \lambda_{2}^{\prime \prime}(0) \delta^{2}+O\left(\delta^{4}\right), \\
\phi(\delta) & =\frac{\pi}{2}+\phi^{\prime}(0) \delta+\frac{1}{6} \phi^{\prime \prime \prime}(0) \delta^{3}+O\left(\delta^{5}\right),
\end{aligned}
$$

and near the pole $\theta=\pi$, we have

$$
\begin{gathered}
E_{1}\left(\lambda_{1}(0)\right) \equiv \frac{\pi}{2}+\phi(\pi-\delta)+\phi^{\prime}(\pi-\delta) \delta=O\left(\delta^{3}\right) \\
E_{2}\left(\lambda_{1}(0)\right) \equiv \lambda_{1}(\pi-\delta)-\lambda_{2}(\pi-\delta)+\delta\left\{\lambda_{1}^{\prime}(\pi-\delta)-\lambda_{2}^{\prime}(\pi-\delta)\right\}=O\left(\delta^{2}\right) .
\end{gathered}
$$

Thus, the initial data $\lambda_{1}(\delta), \lambda_{1}(\delta)$, and $\phi(\delta)$ can be expressed in terms of the single unknown parameter $\lambda_{1}(0)$. The system of ODEs (2.8) can then be solved using a shooting method. The errors $E_{1}$ and $E_{2}$ defined above are thus functions of $\lambda_{1}(0)$. We iterate on $\lambda_{1}(0)$ so that the absolute value of either $E_{1}$ or $E_{2}$ is smaller than a prescribed tolerance value. We have tried both error functions to validate our numerical code. We could also shoot from both poles and match the solutions at $\theta=\pi / 2$, but this would require iteration on two unknowns (namely $\lambda_{1}(0)$ and $\lambda_{1}(\pi)$ ). We decide to use the approach of shooting 
from one pole to the other pole due to its simplicity. The only disadvantage is that the off-target solutions tend to blow up when they reach the other pole, and so manual adjustment is required. In a typical calculation, we evaluate $E_{1}\left(\lambda_{1}(0)\right)$ for a discrete set of $\lambda_{1}(0)$ values from 1 to 15 in steps of 0.1 initially. As $\lambda_{1}(0)$ increases, $E_{1}\left(\lambda_{1}(0)\right)$ would be finite over one interval and then blow up over the next interval. This alternate behaviour may occur a number of times. It is usually the case that $E_{1}\left(\lambda_{1}(0)\right)$ would change sign just before or after it experiences blow-up. The sign change is captured by increasing or decreasing $\lambda_{1}(0)$ in smaller steps. Once a sign change is found, Newton method is then used to find the root more precisely.

We wish to emphasize that by varying $\lambda_{1}(0)$ over the entire range $(1, \infty)$ (of course for most rubber materials, the $\infty$ here can be replaced by 15), this approach enables us to find all possible axi-symmetric solutions at any given pressure value. For the Ogden material model, we find that

(i) For $0<P<P_{\min }$ or $P>P_{\max }$, there is only one solution, corresponding to the single uniform (i.e. spherical) solution;

(ii) For $P_{\min }<P<P_{\mathrm{cr} 2}$ or $P_{\mathrm{cr} 1}<P<P_{\max }$, there are three solutions, corresponding to three uniform solutions with different radii;

(iii) For $P_{\text {cr2 }}<P<P_{\text {cr1 }}$, the interval where bifurcation is possible, there are five solutions, three of them are uniform solutions and the other two are pear-shaped solutions. The two pear-shaped configurations are mirror images of each other with respect to the equatorial plane $\theta=\pi / 2$.

Although higher-mode bifurcated solutions are not excluded by a general bifurcation analysis (Haughton and Ogden 1978) unless additional constitutive assumptions are imposed (Shield 1972), we confirm that they are not possible for the Ogden material model, as has previously been shown by Needleman (1977).

\section{$3 \quad$ Stability under pressure control}

Take any solution of the equilibrium equations (3.3) (that is, either spherical or pearshaped), and denote by $\bar{P}$ the associated pressure. We first consider the stability of such a solution when inflation is pressure controlled. The sum of the strain energy and potential energy, scaled by $2 \pi H$, is given by

$$
E=\int_{0}^{\pi} W\left(\lambda_{1}, \lambda_{2}\right) \sin \theta d \theta-\frac{1}{2} \bar{P} \int_{0}^{\pi} r^{2} z^{\prime} d \theta .
$$

Before proceeding to detailed calculations, we first compare two commonly used stability criteria, and show that they give complementary stability predications. 
To simplify notation, we shall write the above total energy symbolically as

$$
E=\int_{0}^{\pi} L\left(\boldsymbol{u}, \boldsymbol{u}^{\prime}\right) d \theta
$$

where $\boldsymbol{u}=\{r(\theta), z(\theta)\}$. The above-mentioned equilibrium solution associated with pressure $\bar{P}$ will be denoted by $\overline{\boldsymbol{u}}=\{\bar{r}, \bar{z}\}$ (and the corresponding stretches by $\bar{\lambda}_{1}$ and $\bar{\lambda}_{2}$ ). The Euler-Lagrange equations associated with (3.2) are given by

$$
\frac{\partial L}{\partial \boldsymbol{u}}-\left(\frac{\partial L}{\partial \boldsymbol{u}^{\prime}}\right)^{\prime}=0
$$

which can be shown to be equivalent to (2.3) and (2.4). If dynamic effects are taken into account with $\boldsymbol{u}$ allowed to depend on time $t$ as well, the equations of motion can be derived by applying the Principle of Least Action, and are given by

$$
-\frac{\partial L}{\partial \boldsymbol{u}}+\left(\frac{\partial L}{\partial \boldsymbol{u}^{\prime}}\right)^{\prime}=\sin \theta \rho \ddot{\boldsymbol{u}}
$$

where $\rho$ is the material density and a superimposed dot denotes differentiation with respect to time $t$.

To assess the stability of $\boldsymbol{u}=\overline{\boldsymbol{u}}$, we may consider perturbations of the form $\boldsymbol{u}=$ $\overline{\boldsymbol{u}}+\boldsymbol{v} \mathrm{e}^{\gamma t}$, where the mode function $\boldsymbol{v}$ and growth rate $\gamma$ are to be determined. On substituting this expansion into (3.4) and linearizing in terms of $\boldsymbol{v}$, we obtain

$$
\mathcal{J}[\boldsymbol{v}] \equiv S \boldsymbol{v}+R \boldsymbol{v}^{\prime}-\left(R^{T} \boldsymbol{v}+Q \boldsymbol{v}^{\prime}\right)^{\prime}=-\sin \theta \rho \gamma^{2} \boldsymbol{v}
$$

where $S, R$ and $Q$ are matrix functions with components given by

$$
S_{i j}=\left.\frac{\partial^{2} L}{\partial u_{i} \partial u_{j}}\right|_{\boldsymbol{u}=\overline{\boldsymbol{u}}}, \quad R_{i j}=\left.\frac{\partial^{2} L}{\partial u_{i} \partial u_{j}^{\prime}}\right|_{\boldsymbol{u}=\overline{\boldsymbol{u}}}, \quad Q_{i j}=\left.\frac{\partial^{2} L}{\partial u_{i}^{\prime} \partial u_{j}^{\prime}}\right|_{\boldsymbol{u}=\overline{\boldsymbol{u}}},
$$

and $R^{T}$ denotes the transpose of $R$. The differential operator $\mathcal{J}$ defined by $(3.5)_{1}$ is called the Jacobi operator in the calculus of variations literature. The equilibrium solution is said to be (linearly) unstable if the above equations subjected to appropriate boundary conditions, viewed as an eigenvalue problem determining $\rho \gamma^{2}$, have a non-trivial solution with positive $\rho \gamma^{2}$. We note that this spectral method is not suitable for predicting stability (i.e. its converse is definitely untrue) because, for instance, algebraic growth may still be possible even if exponentially growing modes do not exist.

An effective method for predicting stability is the energy criterion by which $\boldsymbol{u}=\overline{\boldsymbol{u}}$ is a stable solution if it is a weak energy minimizer. For conservative systems such as the one under consideration, there is a connection between this energy criterion and nonlinear Liapunov stability (see, e.g., van der Heijden 2009). For sufficiently small variations $\boldsymbol{v}$ (we only consider variations for which $\boldsymbol{v}$ and $\boldsymbol{v}^{\prime}$ are of the same order), we have

$$
E(\overline{\boldsymbol{u}}+\boldsymbol{v})-E(\overline{\boldsymbol{u}})=\frac{1}{2} \delta^{2} E+O\left(|\boldsymbol{v}|^{3}\right),
$$


where the second variation $\delta^{2} E$ is given by

$$
\delta^{2} E=\int_{0}^{\pi}\left(\boldsymbol{v} \cdot S \boldsymbol{v}+2 \boldsymbol{v} \cdot R \boldsymbol{v}^{\prime}+\boldsymbol{v}^{\prime} \cdot Q \boldsymbol{v}^{\prime}\right) d \theta
$$

Under the assumption that $Q$ is positive definite throughout the interval $0<\theta<\pi$ (or equivalently, $\mathcal{J}[\boldsymbol{v}]$ is an elliptic operator), the second variation $\delta^{2} E$ subject to the normalization condition

$$
\int_{0}^{\pi} \sin \theta|\boldsymbol{v}|^{2} d \theta=1
$$

has a minimum. With the use of a standard argument, it can easily be shown that this constrained minimization problem gives rise to the same eigenvalue problem $(3.5)_{2}$ with $\rho \gamma^{2}$ now playing the role of the Lagrangian multiplier associated with the constraint (3.7); see, e.g., Giaquinta and Hilderbrandt (2004, p.269).

On forming the dot product of (3.5) with $\boldsymbol{v}$ and integrating the resulting expression from 0 to $\pi$ by parts, we find that $-\rho \gamma^{2}$ is equal to $\delta^{2} E$. Thus, stability assessment is reduced to the determination of the sign of the smallest eigenvalue of the eigenvalue problem associated with (3.5). The solution $\overline{\boldsymbol{u}}$ is stable if the smallest eigenvalue is positive (by the energy criterion) and unstable if the smallest eigenvalue is negative (by the spectral method). In some studies, a negative minimum of the second variation of the total energy implying instability is simply assumed to be part of the energy stability criterion. In the rest of this paper we do not distinguish whether instability is deduced by the spectral method or by the energy criterion.

We now proceed to detailed calculations. A straightforward calculation shows that the second variation of energy can be reduced to the form

$$
\delta^{2} E=\int_{0}^{\pi}\left(a_{1} v_{1}^{2}+2 a_{2} v_{1} v_{2}^{\prime}+a_{3} v_{2}^{\prime 2}+a_{4} v_{1}^{\prime 2}+2 a_{5} v_{1}^{\prime} v_{2}^{\prime}\right) d Z
$$

where

$$
\begin{aligned}
& a_{1}=W_{11} / R(\theta)-\left(W_{12} r^{\prime} / \lambda_{2}\right)^{\prime}-\bar{P} z^{\prime}, \\
& a_{2}=W_{12} z^{\prime} / \lambda_{2}-\bar{P} r, \\
& a_{3}=R(\theta)\left(\lambda_{2} W_{22}-W_{2}\right) \lambda_{2}^{-3} z^{\prime 2}+R(\theta) W_{2} \lambda_{2}^{-1}, \\
& a_{4}=R(\theta)\left(\lambda_{2} W_{22}-W_{2}\right) \lambda_{2}^{-3} r^{\prime 2}+R(\theta) W_{2} \lambda_{2}^{-1}, \\
& a_{5}=R(\theta)\left(\lambda_{2} W_{22}-W_{2}\right) \lambda_{2}^{-3} r^{\prime} z^{\prime},
\end{aligned}
$$

and the right hand side of (3.9) is evaluated at the equilibrium state $\overline{\boldsymbol{u}}$.

We observe that positive definiteness of the matrix function $Q$ is equivalent to $a_{3}>$ $0, a_{4}>0$, and $a_{3} a_{4}-a_{5}^{2}>0$. These are essentially the two dimensional version of the strong ellipticity condition. We have checked to verify that these inequalities are indeed satisfied for all the inflation solutions that we have found. 
In view of the fact that the integrand in (3.8) does not contain $v_{2}$, we follow Chen (1997) and replace the normalization condition (3.7) by

$$
\int_{0}^{\pi}\left(v_{1}^{2}+v_{2}^{\prime 2}\right) d \theta=1
$$

The advantage of using this normalization is that $v_{2}^{\prime}$ can then be eliminated from the two equations satisfied by $v_{1}$ and $v_{2}^{\prime}$ :

$$
a_{1} v_{1}+a_{2} v_{2}^{\prime}-\left(a_{4} v_{1}^{\prime}+a_{5} v_{2}^{\prime}\right)^{\prime}=\alpha v_{1}, \quad a_{2} v_{1}+a_{3} v_{2}^{\prime}+a_{5} v_{1}^{\prime}=\alpha v_{2}^{\prime},
$$

and we then end up with the simple eigenvalue problem

$$
\left[b_{2} v_{1}^{\prime}\right]^{\prime}+b_{0} v_{1}=0, \quad 0 \leq \theta \leq \pi ; \quad v_{1}(0)=v_{1}(\pi)=0
$$

where

$$
b_{0}=-a_{1}-\left(\frac{a_{2} a_{5}}{a_{3}-\alpha}\right)^{\prime}+\frac{a_{2}^{2}}{a_{3}-\alpha}+\alpha, \quad b_{2}=a_{4}-\frac{a_{5}^{2}}{a_{3}-\alpha},
$$

and $\alpha$ in (3.12) is the Lagrangian multiplier associated with the constraint (3.10). If we were to use the normalization condition (3.7), $v_{1}$ and $v_{2}$ would satisfy

$$
a_{1} v_{1}+a_{2} v_{2}^{\prime}-\left(a_{4} v_{1}^{\prime}+a_{5} v_{2}^{\prime}\right)^{\prime}=\alpha \sin \theta v_{1}, \quad\left(a_{2} v_{1}+a_{3} v_{2}^{\prime}+a_{5} v_{1}^{\prime}\right)^{\prime}=-\alpha \sin \theta v_{2},
$$

from which we would not be able to eliminate $v_{2}$. The two approaches should be equivalent as far as determination of the sign of the smallest eigenvalue is concerned since the normalization used should only affect the absolute value of the eigenvalue, but not its sign. Calculations conducted by Fu and Xie (2010) for a similar problem show that the variations of the smallest eigenvalue with respect to the inflation pressure, obtained from the two approaches, become almost indistinguishable after they are appropriately scaled.

The multiplier $\alpha$ can again be shown to be equal to the value of $\delta^{2} E$. Thus, determination of stability is reduced to finding the sign of the lowest eigenvalue of (3.11). The static solution $\boldsymbol{u}=\overline{\boldsymbol{u}}$ is unstable if the eigenvalue problem (3.11) has at least one negative eigenvalue and is stable if no negative eigenvalues or zero eigenvalue exists. We note that since $a_{3}>0$, the expressions in (3.12) are not singular at least when $\alpha$ is negative. In our numerical calculations, we only seek negative values of $\alpha$ since their existence/non-existence alone is sufficient to establish instability/stability.

When $\alpha=0$ and the coefficients are evaluated at the uniform inflation solution $\lambda_{1}=$ $\lambda_{2}=\lambda$, the eigenvalue problem (3.11) reduces to the bifurcation problem for an adjacent equilibrium. It is known that the mode-one bifurcation solution

$$
v_{1}=\sin \theta \cos \theta
$$

is possible when the bifurcation condition (2.11) is satisfied. We have verified that when (3.13) is assumed, the left hand side of (3.11) does indeed become a multiple of $\lambda\left(W_{11}+\right.$ $\left.W_{12}\right)-W_{1}$. 
We now proceed to solve the eigenvalue problem (3.11). The coefficients in $(3.11)_{1}$ cannot be evaluated at the two poles directly. Thus, as in the previous section, we replace the interval by $[\delta, \pi-\delta]$. By substituting the series solution

$$
v_{1}=v_{1}^{\prime}(0) \theta+\frac{1}{2} v_{1}^{\prime \prime}(0) \theta^{2}+\frac{1}{6} v_{1}^{\prime \prime \prime}(0) \theta^{3} \ldots
$$

into the left hand side of $(3.11)_{1}$ and then equating the coefficients of powers of $\theta$ to zero, we may express $v_{1}^{\prime \prime}(0)$ and other higher order derivatives in terms of the lowest-order derivative $v_{1}^{\prime}(0)$. For instance, we have

$$
\frac{v_{1}^{\prime \prime}(0)}{v_{1}^{\prime}(0)}=-\frac{2 \alpha}{3 W_{11}}
$$

and

$$
\begin{array}{r}
\frac{v_{1}^{\prime \prime \prime}(0)}{v_{1}^{\prime}(0)}=\frac{1}{8 b^{2} \lambda_{1}^{2} W_{11}}\left\{-18 W_{1}\left(a^{2} \lambda_{1}+b^{2} \lambda_{2}^{\prime \prime}-3 b^{2} \lambda_{1}^{\prime \prime}\right)+2 \lambda_{1}^{2} b^{2} \alpha^{2} W_{11}^{-1}\right. \\
+\lambda_{1}^{2} W_{11}\left(30 a^{2}-8 b^{2}\right)+6 a^{2} \lambda_{1}^{2} W_{12}-6 b^{2} \lambda_{1}\left(3 W_{11}+W_{12}\right)\left(3 \lambda_{1}^{\prime \prime}-\lambda_{2}^{\prime \prime}\right) \\
+3 b^{2} \lambda_{1}^{2}\left[W_{111}\left(\lambda_{1}^{\prime \prime}-3 \lambda_{2}^{\prime \prime}\right)-W_{112}\left(5 \lambda_{1}^{\prime \prime}+\lambda_{2}^{\prime \prime}\right)-2 P \sqrt{a^{2} \lambda_{1}^{2}+b^{2} \lambda_{1}\left(\lambda_{2}^{\prime \prime}-3 \lambda_{1}^{\prime \prime}\right)}\right\} .
\end{array}
$$

where the right hand sides are evaluated at the equilibrium state $\overline{\boldsymbol{u}}$ and at $\theta=0$. A similar procedure can be applied at the pole $\theta=\pi$. We find that

$$
\frac{v_{1}^{\prime \prime}(\pi)}{v_{1}^{\prime}(\pi)}=\frac{2 \alpha}{3 W_{11}}
$$

but the expression for $v_{1}^{\prime \prime \prime}(\pi) / v_{1}^{\prime}(\pi)$ has the same form as the right hand side of $(3.15)$ although it is now evaluated at $\theta=\pi$. We note that although the second order equation $(3.11)_{1}$ should have two independent series solutions at each end, only one solution is obtained near each pole after $v_{1}(0)=0$ or $v_{1}(\pi)=0$ has been imposed.

It can easily be deduced that

$$
\frac{v_{1}(\delta)}{v_{1}^{\prime}(\delta)}=V_{L}(\delta)+O\left(\delta^{4}\right), \quad \frac{v_{1}(\pi-\delta)}{v_{1}^{\prime}(\pi-\delta)}=V_{R}(\delta)+O\left(\delta^{4}\right),
$$

where

$$
\begin{gathered}
V_{L}(\delta)=\delta-\frac{v_{1}^{\prime \prime}(0)}{2 v_{1}^{\prime}(0)} \delta^{2}+\left\{\frac{1}{2}\left(\frac{v_{1}^{\prime \prime}(0)}{v_{1}^{\prime}(0)}\right)^{2}-\frac{1}{3} \frac{v_{1}^{\prime \prime \prime}(0)}{v_{1}^{\prime}(0)}\right\} \delta^{3}, \\
V_{R}(\delta)=-\delta-\frac{v_{1}^{\prime \prime}(\pi)}{2 v_{1}^{\prime}(\pi)} \delta^{2}+\left\{-\frac{1}{2}\left(\frac{v_{1}^{\prime \prime}(\pi)}{v_{1}^{\prime}(\pi)}\right)^{2}+\frac{1}{3} \frac{v_{1}^{\prime \prime \prime}(\pi)}{v_{1}^{\prime}(\pi)}\right\} \delta^{3} .
\end{gathered}
$$

We may then solve $(3.11)_{1}$ subject to the initial conditions

$$
v_{1}(\delta)=V_{L}(\delta), \quad v_{1}^{\prime}(\delta)=1
$$

and iterate on $\alpha$ so that the target condition

$$
v_{1}(\pi-\delta)-V_{R}(\delta) v_{1}^{\prime}(\pi-\delta)=0
$$




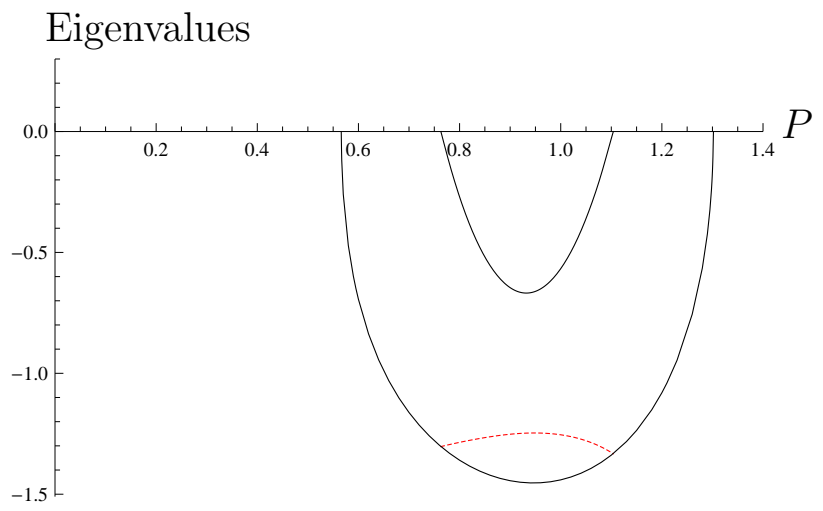

Figure 2: Unstable eigenvalues for a pressurized spherical balloon. Solid lines: eigenvalues associated with the spherical solution, the smaller eigenvalue having been magnified 10 times in order to show it together with the bigger eigenvalue. Dashed line: the single eigenvalue associated with the pear-shaped solution.
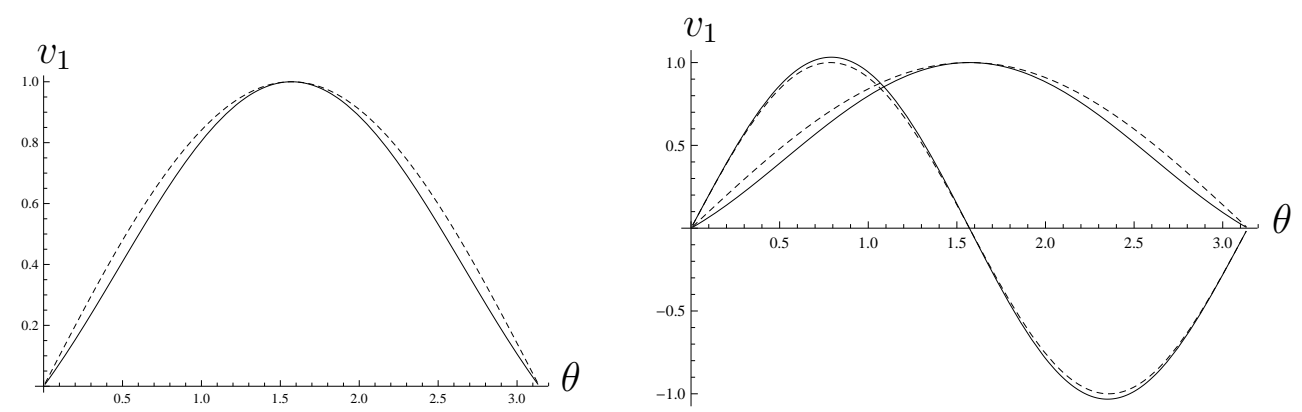

Figure 3: Eigenfunctions associated with the spherical solution. Left: $P=1.2$ for which there is a single unstable mode which is well approximated by $\sin \theta$ (dashed line). Right: $P=1$ for which there are two unstable modes which are well approximated by $\sin \theta$ and $\sin 2 \theta$, respectively (dashed lines).

is satisfied to within a specified tolerance. We have taken $v_{1}^{\prime}(\delta)=1$ without loss of generality since this is a linear problem and the eigenfunction can be normalized in any manner. It is found sufficient to choose $\delta=0.05$.

For the uniform (spherical) solutions on the descending branch of the pressure-stretch curve in Fig. 1, we find that for each pressure value in $\left(P_{\mathrm{cr} 1}, P_{\max }\right)$ or $\left(P_{\min }, P_{\mathrm{cr} 2}\right)$ the eigenvalue problem (3.11) has one negative eigenvalue, whereas for each pressure value in $\left(P_{\mathrm{cr} 2}, P_{\mathrm{cr} 1}\right)$, there are two negative eigenvalues. Existence of an additional eigenvalue reflects the fact that the spherical configuration also has a tendency to move to a pearshaped configuration in order to achieve lower energy. Fig. 2 shows the variation of the eigenvalues over their ranges of existence. When $P=1.2$, the single negative eigenvalue is equal to -1.073 , whereas when $P=1$, there are two negative eigenvalues, given by -1.445 and -0.0612 , respectively. The associated eigenfunctions are shown in Fig. 3. For 
comparison, we have also shown $\sin \theta$ and $\sin 2 \theta$ in dashed lines, the significance of which is as follows: In the limit $P \rightarrow P_{\max }$ the eigenfunction associated with the unique unstable eigenmode would tend to $\sin \theta$ (corresponding to a spherical perturbation), whereas in the limit $P \rightarrow P_{\mathrm{cr} 1}$ or $P_{\mathrm{cr} 2}$, the eigenfunction of the second unstable eigenmode associated with the spherical solution would tend to $\sin 2 \theta$ (corresponding the perturbed configuration adopting a pear shape). In the next section, the first and second modes are referred to as symmetric and anti-symmetric modes, respectively.

For each bifurcated pear-shaped solution that exists for pressure values in $\left(P_{\mathrm{cr} 2}, P_{\mathrm{cr} 1}\right)$, we have also found one negative eigenvalue (shown in dotted line in Fig. 2), and so the pear-shaped configuration is also unstable under pressure control with respect to axisymmetric perturbations.

\section{Stability when the balloon is inflated by an ideal gas}

In this section we consider the more realistic case when the balloon is inflated by an ideal gas. In this case the scaled total energy (3.1) is replaced by

$$
E=\int_{0}^{\pi} W\left(\lambda_{1}, \lambda_{2}\right) \sin \theta d \theta+\phi(V, M)
$$

where

$$
\phi(V, M)=-k M \ln \frac{V}{V_{0}},
$$

with $k$ and $M$ denoting a positive constant of the gas and the total mass of the enclosed gas (scaled by $2 \pi H$ ), and $V_{0}$ the initial internal volume. The pressure $P$ (scaled by $\mu H)$ is computed according to $H P=-\partial(2 \pi H \phi) / \partial V=k(2 \pi H M) / V$. In the case of mass control, the total mass $M$ is fixed, but $P$ and $V$ are allowed to vary subject to the constraint $P V=2 \pi k M$.

We first show that the two stability criteria discussed in the previous section again give complementary stability predictions. To this end, we write (4.1) symbolically as

$$
E=\int_{0}^{\pi} L\left(\boldsymbol{u}, \boldsymbol{u}^{\prime}\right) d \theta+\phi(V, M)+\frac{1}{2} \bar{P} \int_{0}^{\pi} r^{2} z^{\prime} d \theta
$$

where $L$ has the same expression as in the previous section. The equation of motion (3.4) is now replaced by

$$
-\frac{\partial L}{\partial \boldsymbol{u}}+\left(\frac{\partial L}{\partial \boldsymbol{u}^{\prime}}\right)+(P-\bar{P}) r\left(\begin{array}{c}
z^{\prime} \\
-r^{\prime}
\end{array}\right)=\sin \theta \rho \ddot{\boldsymbol{u}},
$$

and the linearized perturbation equation (3.5) by

$$
-S \boldsymbol{v}-R \boldsymbol{v}^{\prime}+\left(R^{T} \boldsymbol{v}+Q \boldsymbol{v}^{\prime}\right)^{\prime}-\frac{\bar{P} \dot{V} \bar{r}}{\bar{V}}\left(\begin{array}{c}
\bar{z}^{\prime} \\
-\bar{r}^{\prime}
\end{array}\right)=\sin \theta \rho \gamma^{2} \boldsymbol{v}
$$


where $\bar{V}$ is the internal volume associated with the equilibrium state $\overline{\boldsymbol{u}}$, and $\dot{V}$ denotes the volume variation given by

$$
\dot{V}=\pi \int_{0}^{\pi}\left(2 \bar{r} \bar{z}^{\prime} v_{1}+\bar{r}^{2} v_{2}^{\prime}\right) d \theta .
$$

Correspondingly, the second variation (3.6) is replaced by

$$
\delta^{2} E=\int_{0}^{\pi}\left(\boldsymbol{v} \cdot S \boldsymbol{v}+2 \boldsymbol{v} \cdot R \boldsymbol{v}^{\prime}+\boldsymbol{v}^{\prime} \cdot Q \boldsymbol{v}^{\prime}\right) d \theta+\phi_{V V} \dot{V}^{2}
$$

where $\phi_{V V}=\partial^{2} \phi /\left.\partial V^{2}\right|_{V=\bar{V}}=\bar{P} /(2 \pi \bar{V})$. Again, it can be shown that minimizing (4.6) subject to the normalization condition (3.7) gives rise to the eigenvalue problem (4.4) with $-\rho \gamma^{2}$ playing the role of the Lagrangian multiplier associated with the normalization condition. Thus, the two stability criteria will give complementary stability predictions as in the previous pressure control case.

We rewrite (4.6) in the form

$$
\delta^{2} E-\phi_{V V} \dot{V}^{2}=\int_{0}^{\pi}\left(a_{1} v_{1}^{2}+2 a_{2} v_{1} v_{2}^{\prime}+a_{3} v_{2}^{\prime 2}+a_{4} v_{1}^{\prime 2}+2 a_{5} v_{1}^{\prime} v_{2}^{\prime}\right) d Z,
$$

where the coefficients $a_{1}, \ldots, a_{5}$ have the same expressions as in the previous section. Since $\phi_{V V}>0$, it is seen immediately that the second variation of potential energy in the current mass control case is always greater than its counterpart in the pressure controlled case, giving rise to the possibility that some of the solutions studied in the previous section may become stable under mass control.

On minimizing the second variation $\delta^{2} E$ subject to the normalization condition (3.10), we obtain the eigenvalue problem

$$
\left[b_{2} v_{1}^{\prime}\right]^{\prime}+b_{0} v_{1}+b_{1} \mathcal{L}\left[v_{1}\right]=0, \quad 0 \leq \theta \leq \pi ; \quad v_{1}(0)=v_{1}(\pi)=0
$$

where

$$
b_{1}=\frac{a_{2} \bar{r}^{2}}{2\left(a_{3}-\alpha\right)}-\frac{1}{2}\left(\frac{a_{5} \bar{r}^{2}}{a_{3}-\alpha}\right)^{\prime}-\bar{r} \bar{z}^{\prime},
$$

and $\mathcal{L}\left[v_{1}\right]$ is a functional of $v_{1}$ defined by

$$
\mathcal{L}\left[v_{1}\right]=\frac{\bar{P} \dot{V}}{\bar{V}}
$$

The expressions for $b_{0}$ and $b_{2}$ are the same as in the pressure control case. After $v_{2}^{\prime}$ is eliminated from the expression for $\dot{V}$, the functional $\mathcal{L}\left[v_{1}\right]$ takes the form

$$
\mathcal{L}\left[v_{1}\right]=-2\left(\frac{\bar{V}}{\pi \bar{P}}+\frac{1}{2} \int_{0}^{\pi} \frac{\bar{r}^{4}}{a_{3}-\alpha} d \theta\right)^{-1} \cdot \int_{0}^{\pi} b_{1} v_{1} d \theta .
$$

Thus, equation $(4.8)_{1}$ with $\mathcal{L}\left[v_{1}\right]$ given by $(4.9)$ is a linear integro-differential equation. We solve the associated eigenvalue problem by considering two cases separately. 
Case 1: $\mathcal{L}\left[v_{1}\right]=0$.

In this case the eigenvalue problem (4.8) is the same as in the pressure control case. However, each eigen solution found in the previous section is a solution for the present mass control case only if the solution also satisfies (4.9) with $\mathcal{L}\left[v_{1}\right]=0$. We note that when $\overline{\boldsymbol{u}}$ corresponds to a spherical solution, the $b_{1}$ is symmetric about the equatorial plane $\theta=\pi / 2$. It then follows that any anti-symmetric mode found in the previous section automatically satisfies (4.9) with $\mathcal{L}\left[v_{1}\right]=0$. Physically, what this means is that the existence of an anti-symmetric mode does not require any change in volume, pressure or mass, and so it is independent of whether the perturbations are pressure, mass, or volume controlled. Thus, we may conclude immediately that the spherical solutions corresponding to $P_{\text {cr2 }}<P<P_{\text {cr1 }}$ are unstable. This is consistent with Chen and Healey's (1991) finding that whenever the pear-shaped configuration were possible, it would have lower total potential energy than the co-existing spherical configuration.

We find that none of the other eigen solutions found in the previous section satisfy (4.9) with $\mathcal{L}\left[v_{1}\right]=0$ and are therefore not solutions of the present eigenvalue problem.

Case 2: $\mathcal{L}\left[v_{1}\right] \neq 0$.

In this case it can easily be verified that (4.8) subject to (4.9) has a nontrivial solution if and only if it has a non-trivial solution when $\mathcal{L}\left[v_{1}\right]=1$ because the eigenvalue problem is linear. Thus, the eigenvalue problem can be reduced to

$$
\left[b_{2} v_{1}^{\prime}\right]^{\prime}+b_{0} v_{1}+b_{1}=0, \quad 0 \leq \theta \leq \pi ; \quad v_{1}(0)=v_{1}(\pi)=0
$$

subject to

$$
2 \int_{0}^{\pi} b_{1} v_{1} d \theta+\left(\frac{\bar{V}}{\pi \bar{P}}+\frac{1}{2} \int_{0}^{\pi} \frac{\bar{r}^{4}}{a_{3}-\alpha} d \theta\right)=0 .
$$

Now (4.10) is no longer an eigenvalue problem: it has a unique solution for any $\alpha$ not equal to an eigenvalue associated with (3.11), and we expect that the unique solution will blow up as an eigenvalue of (3.11) is approached, a fact that will manifest itself in our numerical calculations (this behavior can also be seen explicitly in the simple prototypical problem $\left.v_{1}^{\prime \prime}(\theta)+\alpha^{2} v_{1}(\theta)+\theta(\theta-\pi)=0, v_{1}(0)=v_{1}(\pi)=0\right)$. Our solution strategy is to decrease $\alpha$ in small steps from $\alpha=0$ to $\alpha=\alpha_{\min }$, where $\alpha_{\min }$ is the smallest eigenvalue of (3.11). For each $\alpha$ we solve the boundary value problem (4.10) and then substitute the unique solution into (4.11) to check whether the latter condition is satisfied or not. If it is satisfied, the associated value of $\alpha$ is an eigenvalue of the eigenvalue problem under consideration. We note that no solution can exist for $\alpha<\alpha_{\min }$ because the total energy under mass control is always greater than the total energy under pressure control.

The asymptotic behavior of the unique solution of (4.10) as $\theta \rightarrow 0$ or $\pi$ can be found in the same manner as in the previous section. We find that

$$
\frac{v_{1}^{\prime \prime}(0)}{v_{1}^{\prime}(0)}=-\frac{2 \alpha}{3 W_{11}}, \quad \frac{v_{1}^{\prime \prime}(\pi)}{v_{1}^{\prime}(\pi)}=\frac{2 \alpha}{3 W_{11}},
$$




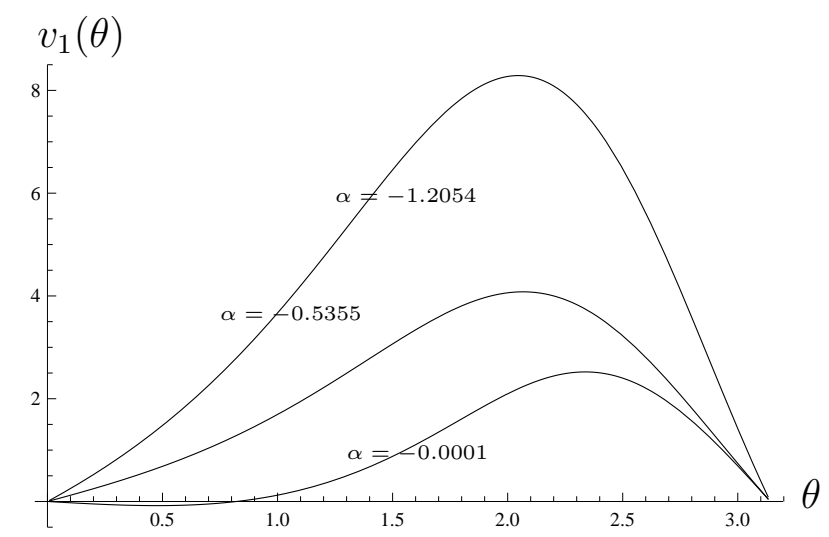

Figure 4: Solutions of (4.10) for the pear-shaped configuration when $\bar{P}=1$. The solution associated with $\alpha=-1.2054$ has been scaled by 10 .

$$
\begin{gathered}
\frac{v_{1}^{\prime \prime \prime}(0)}{v_{1}^{\prime}(0)}=\frac{1}{8 \lambda_{1}^{2} W_{11}}\left\{-18 W_{1}\left(\lambda_{1}+\lambda_{2}^{\prime \prime}-3 \lambda_{1}^{\prime \prime}\right)+2 \lambda_{1}^{2} \alpha^{2} W_{11}^{-1}+22 \lambda_{1}^{2} W_{11}\right. \\
+6 \lambda_{1}^{2} W_{12}-6 \lambda_{1}\left(3 W_{11}+W_{12}\right)\left(3 \lambda_{1}^{\prime \prime}-\lambda_{2}^{\prime \prime}\right)+3 \lambda_{1}^{2}\left[W_{111}\left(\lambda_{1}^{\prime \prime}-3 \lambda_{2}^{\prime \prime}\right)-W_{112}\left(5 \lambda_{1}^{\prime \prime}+\lambda_{2}^{\prime \prime}\right)\right] \\
\left.-6 \lambda_{1}^{2}\left(\bar{P}-\frac{\lambda_{1}}{v_{1}^{\prime}(0)}\right) \sqrt{a^{2} \lambda_{1}^{2}+\lambda_{1}\left(\lambda_{2}^{\prime \prime}-3 \lambda_{1}^{\prime \prime}\right)}\right\} \\
\frac{v_{1}^{\prime \prime \prime}(\pi)}{v_{1}^{\prime}(\pi)}=\frac{1}{8 \lambda_{1}^{2} W_{11}}\left\{-18 W_{1}\left(\lambda_{1}+\lambda_{2}^{\prime \prime}-3 \lambda_{1}^{\prime \prime}\right)+2 \lambda_{1}^{2} \alpha^{2} W_{11}^{-1}+22 \lambda_{1}^{2} W_{11}\right. \\
+6 \lambda_{1}^{2} W_{12}-6 \lambda_{1}\left(3 W_{11}+W_{12}\right)\left(3 \lambda_{1}^{\prime \prime}-\lambda_{2}^{\prime \prime}\right)+3 \lambda_{1}^{2}\left[W_{111}\left(\lambda_{1}^{\prime \prime}-3 \lambda_{2}^{\prime \prime}\right)-W_{112}\left(5 \lambda_{1}^{\prime \prime}+\lambda_{2}^{\prime \prime}\right)\right] \\
\left.-6 \lambda_{1}^{2}\left(\bar{P}+\frac{\lambda_{1}}{v_{1}^{\prime}(\pi)}\right) \sqrt{a^{2} \lambda_{1}^{2}+\lambda_{1}\left(\lambda_{2}^{\prime \prime}-3 \lambda_{1}^{\prime \prime}\right)}\right\}
\end{gathered}
$$

where of course the right hand sides of (4.12) - (4.14) are evaluated at the equilibrium solution $\overline{\boldsymbol{u}}$ and at the corresponding pole.

The asymptotic expansions for $v_{1}(\delta) / v_{1}^{\prime}(\delta)$ and $v_{1}(\pi-\delta) / v_{1}^{\prime}(\pi-\delta)$ take the same form as in (3.16)-(3.18). The boundary value problem (4.10) is then solved using the following shooting procedure. We first make a guess for $v_{1}^{\prime}(\delta)$ and compute $v_{1}(\delta)$ according to $v_{1}^{\prime}(\delta)=V_{L}(\delta) v_{1}^{\prime}(\delta)$. Equation (4.10) is then integrated from $\theta=\delta$ to $\theta=\pi-\delta$. We iterate on $v_{1}^{\prime}(\delta)$ so that the target condition

$$
v_{1}^{\prime}(\pi-\delta)-V_{R}(\delta) v_{1}^{\prime}(\pi-\delta)=0
$$

is satisfied. In evaluating (4.13) and (4.14) we may replace $v_{1}^{\prime}(0)$ and $v_{1}^{\prime}(\pi)$ in the last terms by $v_{1}^{\prime}(\delta)$ and $v_{1}^{\prime}(\pi-\delta)$, respectively. We note that the left hand side of (4.15) is of order $\delta^{4}$ and so typically we set the iteration to stop when the absolute value of the left hand side is less than $\delta^{4}$.

Implementation of the above numerical procedure on the Mathematica platform (Wolfram 1991) is straightforward. In Fig. 4, we have shown solutions associated with the pear-shaped configuration when $\alpha=-0.0001,-0.5355,-1.2054$, respectively. It is seen 

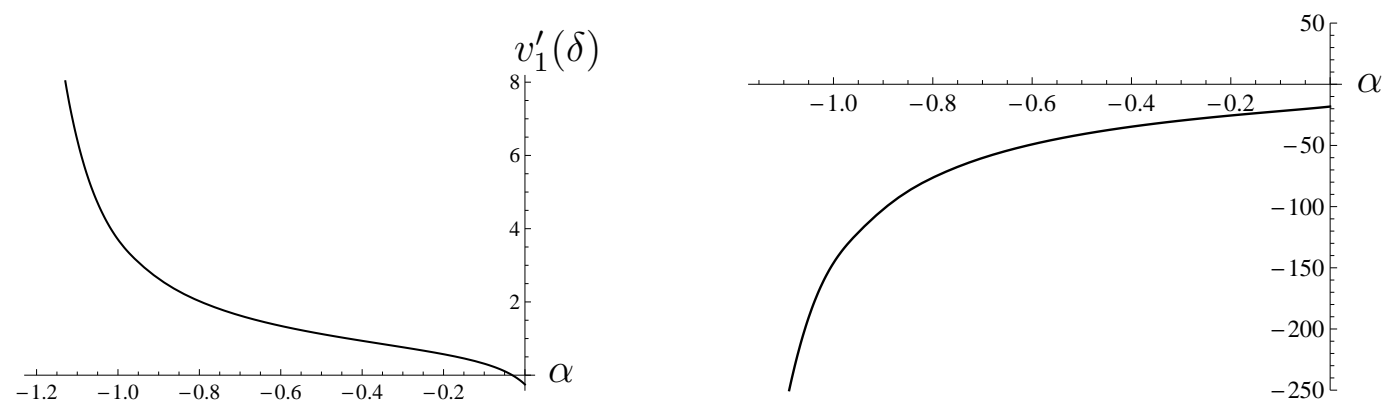

Figure 5: A typical set of results for the pear-shaped configuration when $\bar{P}=1$. Left: Variation of $v_{1}^{\prime}(\delta)$ with respect to $\alpha$. Right: Variation of the left hand side of (4.11) with respect to $\alpha$.
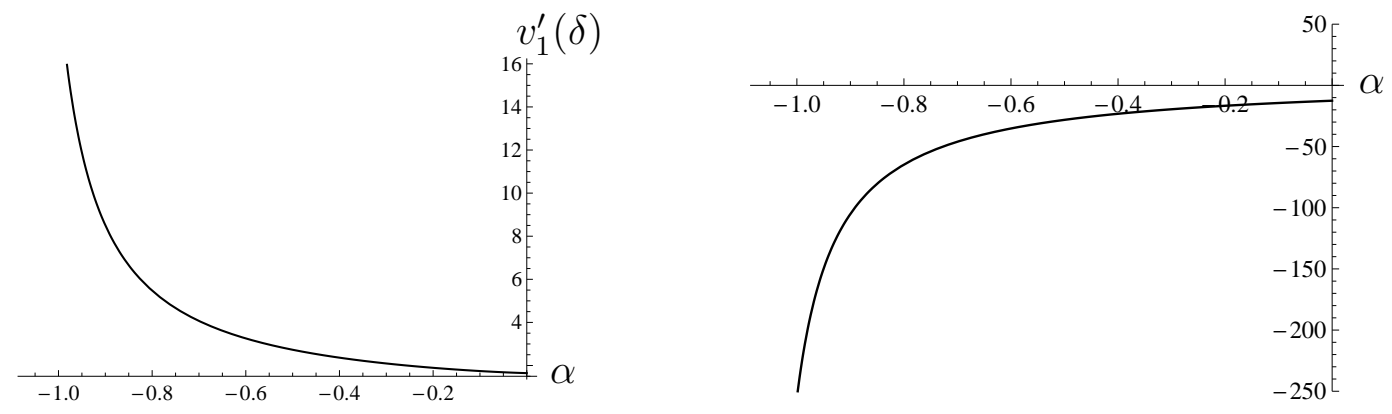

Figure 6: A typical set of results for the spherical configuration when $\bar{P}=1.2$. Left: Variation of $v_{1}^{\prime}(\delta)$ with respect to $\alpha$. Right: Variation of left hand side of (4.11) with respect to $\alpha$.

that the solution does blow up, as remarked earlier, when $\alpha$ approaches $\alpha_{\text {min }}$ which is approximately -1.25 when $\bar{P}=1$. For pear-shaped configurations which can only exist for $P_{\text {cr2 }}<\bar{P}<P_{\text {cr1 }}$, we find that a solution of (4.10) that also satisfies (4.11) does not exist. In Fig. 5 we have shown a typical calculation corresponding $\bar{P}=1$ : the two figures show variations of $v_{1}^{\prime}(\delta)$ and the left hand side of (4.11) with respect to $\alpha$, respectively. The figure on the right shows that (4.11) is never satisfied.

For spherical configurations, we only need to examine their stability for values of $\bar{P}$ outside the interval $\left(P_{\mathrm{cr} 2}, P_{\mathrm{cr} 1}\right)$ since in the latter interval instability has already been demonstrated. Again, solutions of (4.10) that also satisfy (4.11) do not exist. In Fig. 6 we have shown a typical calculation corresponding $\bar{P}=1.2$.

We thus conclude that under mass control, a pear-shaped configuration is stable whenever it exists, and that each spherical configuration is stable when an adjacent pear-shaped configuration does not exist and is unstable otherwise. 


\section{Conclusion}

Stability of a pressurized membrane balloon is an important fundamental problem since in a variety of applications it is necessary to determine whether a pressurized membrane balloon is stable or not due to the fact that only stable configurations can be observed/realized. In this paper we considered the simplest form of a pressurized balloon - a balloon which is initially spherical. We determined the stability properties of the spherical and pear-shaped configurations that can exist on the descending branch of the pressure versus stretch/volume curve. The stability is determined with respect to general axi-symmetric perturbations. We showed that the pear-shaped configuration that bifurcates from the spherical configuration is stable under mass control but unstable under pressure control. This is consistent with the experimental observations made by Alexander (1971). We remark that although our calculations are carried out for the Ogden material model, we expect that the qualitative stability behaviour is also valid for other material models that predict a similar $N$-shaped behaviour for uniform inflation.

Previous stability studies have mostly been concerned with spherical perturbations. The associated results can be recovered from our general formulation as follows. For a uniformly inflated configuration corresponding to $\lambda_{1}=\lambda_{2}=\lambda$, if the variations are spherical such that $v_{1}=\sin \theta, v_{2}=-\cos \theta$ (we take the amplitude to be unity without loss of generality), then the second variations (3.8) and (4.6) reduce, respectively, to

$$
\delta^{2} E=4\left(W_{11}+W_{12}-\bar{P} \lambda\right)=2 \lambda^{2} \frac{d \bar{P}}{d \lambda},
$$

and

$$
\delta^{2} E=2\left(2 W_{11}+2 W_{12}+\bar{P} \lambda\right)=2 \lambda^{2}\left\{\frac{d \bar{P}}{d \lambda}-\left.\frac{d \bar{P}_{\text {gas }}}{d \lambda}\right|_{\text {fixed M }}\right\},
$$

where $\bar{P}$ is equal to the $P$ given by $(2.10)_{4}, \bar{P}_{\text {gas }}$ is given by $\bar{P}_{\text {gas }}=k M / V$ with $V=4 \lambda^{3} / 3$, and we have $\bar{P}=\bar{P}_{\text {gas }}$ at any particular equilibrium state. On the descending branch of the pressure versus stretch curve, $d \bar{P} / d \lambda$ is negative, but $d \bar{P}_{\text {gas }} / d \lambda$ is usually more negative than $d \bar{P} / d \lambda$ (Alexander 1971). This is the basis on which each uniform solution associated with the descending branch was thought to be unstable under pressure control but stable under mass control. However, this simple argument fails to predict the instability of the spherical configuration whenever an adjacent pear-shaped configuration is possible.

Finally, we remark that although we have not discussed the spherical solutions associated with the two ascending branches of the pressure versus stretch curve, a few sample calculations using our methodology do confirm the usual expectation that these configurations are stable under both pressure and mass controls. 


\section{Acknowledgements}

This work is supported by the National Science Foundation of China (Grant Nos 11372212 and 11172201).

\section{References}

[1] Adkins, J.E., Rivlin, R.S., 1952. Large elastic deformations of isotropic materials, IX. The deformation of thin shells. Trans. roy. Soc. Lond. A 244, 505-531.

[2] Alexander, H., 1971. Tensile instability of initially spherical balloons. Int. J. Eng. Sci. 9, 151-162.

[3] Austin, G.M., Schievink, W., Williams, R., 1989. Controlled pressure-volume factors in the enlargement of intracranial aneurysms. Neurosurgery 24, 722-730.

[4] Chen, Y-C., 1997. Stability and bifurcation of finite deformations of elastic cylindrical membranes - part I. stability analysis. Int. J. Solids Struct. 34, 1735-1749.

[5] Chen, Y.-C., Healey, T., 1991. Bifurcation to pear-shaped equilibria of pressurized spherical membranes. Int. J. Nonlinear Mech. 26, 279-291.

[6] Crisp, J.D.C. and Hart-Smith, L.J., 1971. Multi-lobed inflated membranes: their stability under finite deformation. Int. J. Solids Struct. 7, 843-861.

[7] De Tommasi, D., Puglisi, G. and Zurlo, G., 2013. Inhomogeneous spherical configurations of inflated membranes. Continuum Mech. Thermodyn. 25, 197-206.

[8] Feodosev, V.I., 1968. On equilibrium modes of a rubber spherical shell under internal pressure. PMM 32, 339-344.

[9] Fu, Y.B., Xie, Y.X., 2010. Stability of localized bulging in inflated membrane tubes under volume control, Int. J. Eng. Sci. 48, 1242-1252.

[10] Giaquinta, M. and Hildebrandt, S., 2004. Calculus of Variations I (2nd Ed), SpringerVerlag, Berlin.

[11] Haughton, D.M., 1980. Post-bifurcation of perfect and imperfect spherical elastic membranes, Int. J. Solids Struct. 16, 1123-1133.

[12] Haughton, D.M. and Ogden, R.W., 1978. On the incremental equations in non-linear elasticity - II. Bifurcation of pressurized spherical shells, J. Mech. Phys Solids 26, 111-138. 
[13] Jenkins, C.H., 2001. Gossamer spacecraft: membrane and inflatable structures technology for space applications, AIAA.

[14] Keplinger, C., Li, T., Baumgartner, R. Suo, Z., and Bauer, S., 2012. Harnessing snapthrough instability in soft dielectrics to achieve giant voltage-triggered deformation, Soft Matter 8, 285-288.

[15] Knops, R.J., 2001. Elements of elastic stability theory. In Topics in Finite Elasticity (eds M. Hayes and G. Saccomandi), CISM Courses and Lectures No. 424, Springer, New York, 169-230.

[16] Marsden, J.E. and Hughes, T.J.R., 1993. Mathematical Foundations of Elasticity, Dover.

[17] Müller, I. and Struchtrup, H., 2002. Inflating a rubber balloon, Math. Mech. Solids $7,569-577$.

[18] Needleman, A., 1977. Inflation of spherical rubber balloons, Int. J. Solids Struct. 13, 409-421.

[19] Ogden, R.W., 1972 Large deformation isotropic elasticity-on the correlation of theory and experiment for incompressible rubber-like solids, Proc. R. Soc. Lond. A326, 565584.

[20] Rudykha, S., Bhattacharya, K. and de Botton, G., 2012. Snap-through actuation of thick-wall electroactive balloons, Int. J. Non-linear Mech. 47, 206C209.

[21] Sagiv, A., 1990. Inflation of an axisymmetric membrane: stress analysis, ASME J. Appl. Mech. 57, 682-687.

[22] Shield, R.T., 1972. On the stability of finitely deformed elastic membranes; Part II: Stability of inflated cylindrical and spherical membranes, ZAMP 23, 16-34.

[23] van der Heijden, A.M.A., 2009. W.T. Koiter's Elastic Stability of Solids and Structures. Cambridge University Press, Cambridge.

[24] Wolfram, S., 1991. Mathematica: A System for Doing Mathematics by Computer (2nd Edn), California, Addison-Wesley.

[25] Youda, M. and Konishi, S., 2002. Acoustic impedance control through structural tuning by pneumatic balloon actuators, Sensors and actuators A95, 222-226. 4, 351403. 